\title{
Asymptomatic Dialysate Turbidity and Repeated Intraductal Clots in a Peritoneal Dialysis Patient
}

\author{
Tian Xu Jingyuan Xie Weiming Wang Hong Ren Nan Chen \\ Department of Nephrology, Institute of Nephrology, Ruijin Hospital, Shanghai Jiao Tong \\ University School of Medicine, Shanghai, China
}

\section{What Is It about?}

- This case report is about a peritoneal dialysis patient who had a constant turbid peritoneal dialysis effluent without abdominal pain. Repeated routine tests of peritoneal effluent samples showed an elevated white blood cell count and an increased number of eosinophils. All microbial cultures of the peritoneal dialysis effluent were negative. However, catheter dysfunction occurred, likely due to a clot forming inside the catheter. Through the pathological examination, this patient was finally diagnosed with fungal peritonitis.

\section{Keywords}

Peritonitis · Eosinophils $\cdot$ Fungus $\cdot$ Asymptomatic $\cdot$ Peritoneal dialysis

\begin{abstract}
A female patient underwent peritoneal dialysis for 2.5 years. She was found to have a constant turbid peritoneal dialysis effluent for 3 months without abdominal pain. Repeated routine tests of peritoneal effluent samples showed an elevated white blood cell count and an increased number of eosinophils. Additionally, a small black spot was found inside the Tenckhoff catheter. The presence of bacteria and fungi was negative by peritoneal fluid culture. Idiopathic eosinophilic peritonitis was considered, and oral corticosteroid treatment was administered. Soon after, the dialysis effluent became clear. However, eosinophilic peritonitis immediately relapsed after the corticosteroid therapy was suspended. Even worse, catheter dysfunction occurred, likely due to a clot that gradually formed from the spot. Finally, the catheter was removed, and a pathological examination was performed. It was revealed that the clot was composed of fungal spores and hyphae as well as eosinophils. This case reminds us that fungal peritonitis should be suspected when a clot forms.




\section{Established Facts}

- It is very rare for a clot to form in a peritoneal dialysis catheter. In particular, when asymptomatic dialysate turbidity and repeated intraductal clots appear at the same time in a peritoneal dialysis patient, what is the correct diagnosis?

\section{Novel Insights}

- This case reminds us that fungal peritonitis should be suspected when a clot forms.

\section{Introduction}

Cloudy peritoneal effluent and abdominal pain are common in peritoneal dialysis (PD)related peritonitis [1]. However, unlike infectious peritonitis, asymptomatic fluid turbidity occasionally occurs in initial PD patients who have an allergy to the dialysate or other components of the dialysis system [2]. This phenomenon is characterized by increased numbers of eosinophils in the effluent of these patients. Therefore, they could also be diagnosed as idiopathic eosinophilic peritonitis (iEP) [2,3]. Catheter dysfunction is another major complication of PD, which leads to technique failure in PD patients [4]. The main causes of catheter dysfunction include catheter migration, omental wrapping, and fibrin blockage [5]. However, intraductal clot has rarely been reported as a cause of catheter malfunction. Here, we report a rare case of simultaneous eosinophilic peritonitis (EP) and intraductal clots.

\section{Case Report}

A 43-year-old woman developed end-stage renal disease due to chronic glomerulonephritis and underwent surgery to insert a Tenckhoff PD catheter in January 2012. She weighed $65 \mathrm{~kg}$ and measured $165 \mathrm{~cm}$ (BMI 23.8). The blood pressure was controlled in the ideal range (130-40/80-90 mm Hg), with a heart rate of approximately $80 \mathrm{bpm}$. Her dialysis scheme was strictly according to the standard continuous ambulatory PD (CAPD), including $2 \mathrm{~L}$ of $1.5 \%$ PD solution with three exchanges daily and $2 \mathrm{~L}$ of $2.5 \%$ PD solution overnight. The ultrafiltration volume was 500-800 mL, and urine output was $800-1,000 \mathrm{~mL} / 24 \mathrm{~h}$. She denied any history of allergic disease, including bronchial asthma or food sensitivity. In general, her CAPD therapy had gone well over the past 2 years, and no PD-related peritonitis had ever occurred.

The woman presented cloudy peritoneal effluent without any symptoms from May 2014. The effluent cytological test and microorganism culture were performed before the administration of antibiotics. The cytological test result showed a karyocyte count of $630 \times 10^{6} / \mathrm{L}$ with $80 \%$ polynuclear cells; however, the microorganism culture was negative. Despite a diagnostic antibiotic treatment with an intraperitoneal injection of cefazolin $(250 \mathrm{mg} / \mathrm{change})$ combined with amikacin ( $25 \mathrm{mg} /$ change) for 2 weeks, the effluent remained turbid. Thence, the antibiotic regimen was changed to intraperitoneal injection of ceftazidime and vancomycin for 21 days. However, there was no improvement in the turbidity. 
Fig. 1. Cytological analysis of the dialysis effluent.

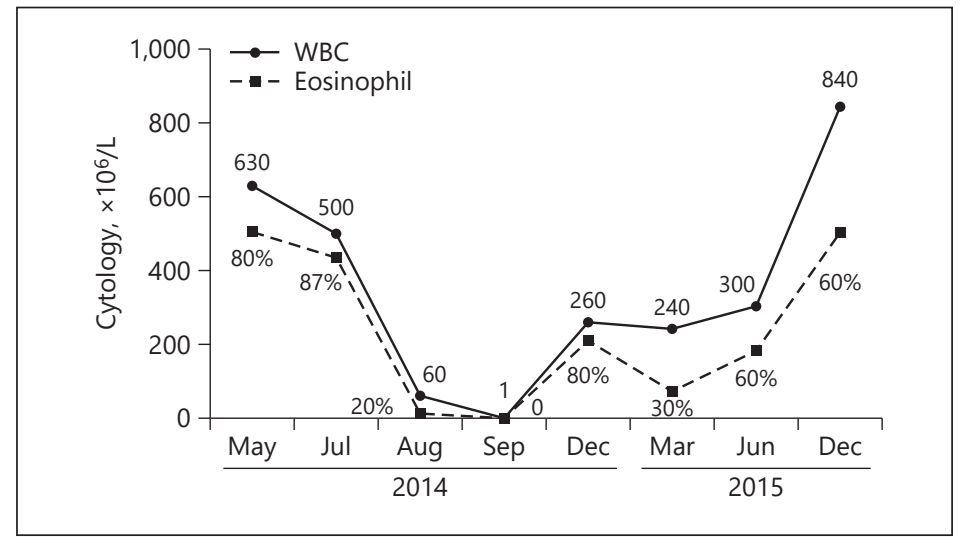

Table 1. Laboratory analysis

\begin{tabular}{lrrrrrrrr}
\hline & $\begin{array}{l}2014 \\
\text { May }\end{array}$ & $\begin{array}{l}\text { 2014 } \\
\text { Jul }\end{array}$ & $\begin{array}{l}2014 \\
\text { Aug }\end{array}$ & $\begin{array}{l}\text { Sep } \\
\text { Se14 }\end{array}$ & $\begin{array}{l}\text { 2015 } \\
\text { Mar }\end{array}$ & $\begin{array}{l}\text { Jun } \\
\text { Jun }\end{array}$ & $\begin{array}{l}\text { Sep } \\
\text { Sep }\end{array}$ & $\begin{array}{l}2015 \\
\text { Dec }\end{array}$ \\
\hline WBCs, $\times 10^{9} / \mathrm{L}$ & 7.3 & 3.8 & 8.99 & 9.3 & 9.5 & 7.9 & 8.7 & 5.4 \\
Eosinophils, \% & 12.8 & 32.2 & 1.3 & 1.1 & 12.4 & 16.7 & 13 & 21.4 \\
Hemoglobin, g/L & 112 & 100 & 91 & 120 & 115 & 113 & 100 & 78 \\
Platelets, $\times 10^{9} / \mathrm{L}$ & 217 & 202 & 242 & 279 & 262 & 282 & 298 & 277 \\
ALT, IU/L & 14 & 15 & 22 & 18 & 16 & 14 & 13 & 6 \\
AST, IU/L & 17 & 16 & 18 & 17 & 14 & 11 & 9 & 12 \\
Albumin, g/L & 28 & 20 & 17 & 25 & 29 & 26 & 25 & 26 \\
Creatinine, mmol/L & 897 & 856 & 816 & 769 & 805 & 902 & 991 & 901 \\
ESR, mm/h & 38 & 42 & 44 & 56 & $\mathrm{ND}$ & 62 & 78 & 110 \\
hs-CRP, mg/L & 1.12 & 0.45 & $\mathrm{ND}$ & 1.33 & 0.57 & 1.41 & $\mathrm{ND}$ & 2.87 \\
Dialysate culture & $\mathrm{N}$ & $\mathrm{N}$ & $\mathrm{N}$ & $\mathrm{N}$ & $\mathrm{N}$ & $\mathrm{N}$ & $\mathrm{N}$ & $\mathrm{N}$ \\
\hline
\end{tabular}

N, Negative; ND, no data.

Therefore, the patient was hospitalized in July 2014. After admission, a careful physical examination showed no rash or purpura. Abdominal palpation revealed no tenderness, tension, or rebound pain. The only abnormal finding was a small black spot adhering inside the external part of the Tenckhoff catheter. The blood routine examination showed a white blood cell count of $3.8 \times 10^{9} / \mathrm{L}$, with $32.2 \%$ eosinophils. There were $500 \times 10^{6} / \mathrm{L}$ nucleated cells in the peritoneal effluent, with $87 \%$ eosinophils. The effluent culture still showed no growth of bacteria or fungi, and it was negative for the acid-fast stain. Serum tumor markers, serum immunological markers such as antinuclear antibody, extractable nuclear antigen, and antineutrophil cytoplasmic antibody, and blood allergen tests were all negative. Enhanced computed tomography did not reveal any signs of malignancy in the chest or abdomen. Additionally, there was a slight elevation in the erythrocyte sedimentation rate (Table 1). As the patient remained asymptomatic, the antibiotic treatment was then stopped. Given that there was a significant elevation of eosinophils in the peritoneal effluent, with a ratio of $87 \%$ and an absolute eosinophil count of $>30 / \mathrm{mm}^{3}$ (up to $435 / \mathrm{mm}^{3}$ ), we initially diagnosed the patient with iEP [6]. Cytological and etiological examinations, including peripheral blood and effluent cell count, effluent bacteria, fungi, and Mycobacterium tuberculosis detection and cultivation, were then repeated several times. (Table 1; Fig. 1).

After a diagnosis of iEP, ketotifen $(1 \mathrm{mg} /$ day) was administered to the patient along with a low-dose prednisone $(20 \mathrm{mg} /$ day $)$ therapy. One day later, the effluent became clear. The 


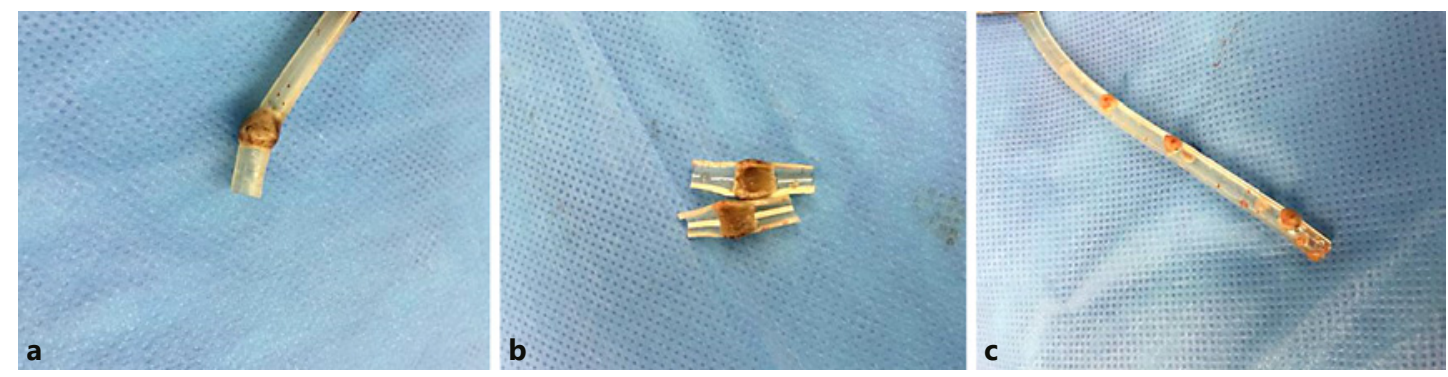

Fig. 2. Intraductal clots. a Clot inside the external Tenckhoff catheter. b Section of clot inside the external Tenckhoff catheter. c Clots inside the internal Tenckhoff catheter.

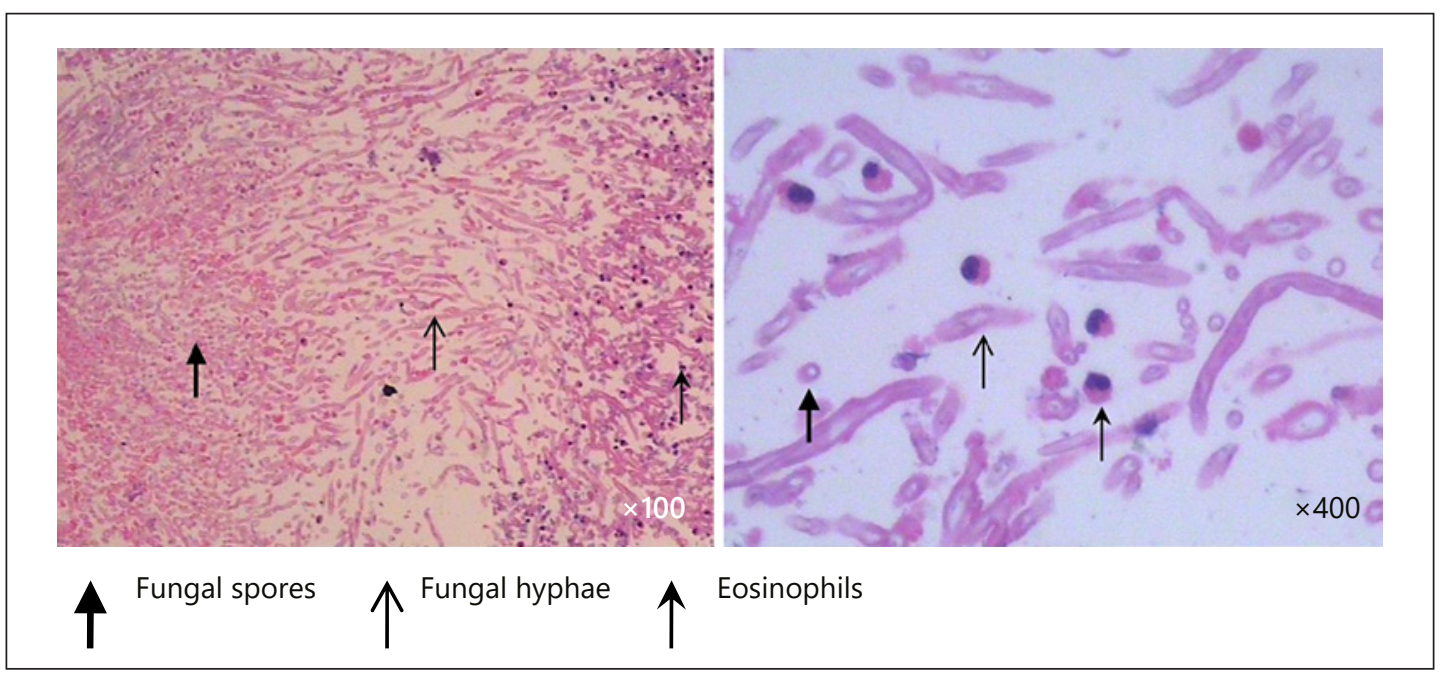

Fig. 3. Pathological examination of the clot.

oral corticosteroid was rapidly reduced and stopped within 2 weeks, but the effluent immediately became turbid again after the suspension of prednisone. We therefore started another course of prednisone (still $20 \mathrm{mg} /$ day), which was tapered and stopped over an 8-week period. During the treatment, she did not feel any abdominal pain and the effluent was still clear. In September 2014, the effluent cytological test showed the polynuclear cell count had decreased to $1 \times 10^{6} / \mathrm{L}$, with no eosinophils (Fig. 1).

Steroid treatment was terminated because of the adverse effects of prednisone, including insomnia and gastrointestinal discomfort. As we expected, the turbid effluent reappeared. For personal reasons, she refused the suggestion of transferring to hemodialysis. PD therapy was continued without any complaints of discomfort. The white blood cell count in the effluent throughout varied within the range of $200-300 \times 10^{6} / \mathrm{L}$ (Fig. 1). It was notable that the small black spot inside the catheter gradually grew larger. In June 2015, the speck ultimately formed a clot, which almost completely blocked the catheter and caused catheter dysfunction. Therefore, we had to remove the external portion of the Tenckhoff catheter.

After surgery, the patient continued PD therapy for 6 months, but the situation gradually reoccurred. Another clot formed (Fig. 2), and catheter dysfunction occurred again. The leukocytes in the peritoneal effluent increased to $8.4 \times 10^{8} / \mathrm{L}$ with $60 \%$ eosinophils, although she was still asymptomatic. After the patient agreed to transfer to hemodialysis, the Tenckhoff catheter was removed immediately. During surgery, we found that there were several clots 
Table 2. Differential diagnosis of eosinophilic peritonitis

\begin{tabular}{|c|c|c|}
\hline & iEP & Secondary EP \\
\hline Common cause & $\begin{array}{l}\text { hypersensitivity to peritoneal } \\
\text { dialysis materials }\end{array}$ & $\begin{array}{l}\text { Mycobacterium tuberculosis } \\
\text { infection, fungal infection }\end{array}$ \\
\hline Onset time & within the first 3 months of PD & after the first 3 months of PD \\
\hline Associated symptoms & rash, itching & abdominal pain, fever \\
\hline Treatment & anti-allergy & anti-infective \\
\hline
\end{tabular}

inside the internal catheter as well (Fig. 2). The removed catheter was sent for pathological examination and bacterial/fungal culture. To our surprise, the pathological examination revealed that the clots consisted of innumerable fungal spores and hyphae as well as eosinophils (Fig. 3). Although the microorganism culture of the catheter was still negative, the patient was eventually diagnosed with EP secondary to fungal infection.

\section{Discussion}

PD-related EP was first reported in 1967 [7]. It is defined as the presence of $>100$ eosinophils $/ \mathrm{mm}^{3}$ or $>10 \%$ eosinophils of the total nonerythrocyte count in the PD effluent [8]. The incidence has been reported to range from 16 to 30\% in intermittent PD patients and is as high as 60\% in CAPD patients [9]. Although Fontán etal. [10] uncovered 5 cases who developed idiopathic EP after $>6$ months and as late as after 63 months, it usually occurs within the first 3 months after initiating PD [11]. Another retrospective study of 112 CAPD patients showed the incidence of EP was $4.4 \%$ and was associated with peripheral blood eosinophilia in $80 \%$ of the patients [12]. Similar to the aforementioned report, eosinophils were increased in both the dialysate and peripheral blood in this case. The typical clinical manifestations of EP include turbid peritoneal effluents, abdominal pain, or fever. It rarely manifest as a pruritic maculopapular rash, arthritis, or expiratory wheezing [13]. In contrast with other cases, this case showed only asymptomatic dialysate turbidity and no symptoms or signs of allergy.

According to the etiology, EP can be divided into iEP and secondary EP (Table 2). The causes and treatments of the two types differ from each other. iEP tends to occur within the first 3 months of PD initiation and is attributed to hypersensitivity to PD materials, drugs, icodextrin, air introduced into the peritoneal cavity at the time of catheter placement, or the mechanical irritant action of large volumes of fluid on the peritoneum. iEP patients usually have a history of allergy, and some allergies may be associated with rash or itching. Unlike the classic clinical manifestation of $\mathrm{iEP}$, in the present case the patient had undergone CAPD for more than 2.5 years without any signs or symptoms of allergy. It appeared that the patient was more likely to have secondary rather than primary EP.

EP can also be proximate to several causes, such as intraperitoneal administration of drugs [14], M. tuberculosis infection [15], and especially fungal infection [16]. Fontán et al. [10] demonstrated that about 7.1\% of EP cases were caused by fungal infections. Similar to a report by Lee and Huang [16], repeated microorganism cultures were negative, and fungal peritonitis was finally diagnosed by a microscopic examination of the clots. One possible reason is that the fungus may have been defective due to the eosinophil attack. The lack of local and systemic symptoms suggested the low virulence of the causative fungi.

The natural course of iEP appears to be self-limiting and resolves spontaneously after several weeks. Although iEP does not require treatment, it can benefit from short courses of therapy with steroids [9]. If there is concern about the side effects of steroids, orally admin- 
istered ketotifen [17] or diphenhydramine [9] are also good choices. Because of the negative results of repeated microorganism cultures, the case was misdiagnosed initially as iEP [6]. The patient was finally diagnosed with fungal peritonitis by microscopic examination, although prednisone was temporarily effective. This serves to remind us that even though oral steroid treatment is effective, EP secondary to microbial infection should still be excluded. Based on the guidelines [1] for the treatment of fungal peritonitis, the Tenckhoff catheter was ultimately removed.

In summary, we have described a patient who developed a delayed and prolonged EP due to a catheter-colonized fungal infection. We propose that EP secondary to fungal infection should be considered, even when repeated microorganism cultures are negative or steroid treatment is effective. The catheter should be removed and sent for microscopic fungal examination immediately once a clot is found inside the catheter.

\section{Statement of Ethics}

The patient provided a written informed consent to allow us to report this case.

\section{Disclosure Statement}

The authors declare that they have no conflict of interest.

\section{Funding Sources}

This study was subsidized by the National Natural Science Foundation of China (No. 81300633) and Shanghai Municipal Commission of Health and Family Planning (No. 20134Y101).

\section{Author Contributions}

T. Xu drafted the manuscript, with the help of H. Ren and N. Chen. J. Xie collected the results of the laboratory tests. W. Wang provided this patient. T. Xu, H. Ren, and N. Chen participated in the patient's diagnosis and treatment. All authors read and approved the final version of the paper. H. Ren takes responsibility for the presentation of this case with honest, accurate, and transparent reporting.

\section{References}

1 Li PK, Szeto CC, Piraino B, Bernardini J, Figueiredo AE, Gupta A, et al.; International Society for Peritoneal Dialysis. Peritoneal dialysis-related infections recommendations: 2010 update. Perit Dial Int. 2010 Jul-Aug; 30(4):393-423.

2 Asghar R, Woodrow G, Turney JH. A case of eosinophilic peritonitis treated with oral corticosteroids. Perit Dial Int. 2000 Sep-Oct;20(5):579-80.

3 Ikee R, Oka M, Maesato K, Mano T, Moriya H, Ohtake T, et al. Eosinophilic peritonitis and ultrafiltration failure on initiation of CAPD. Perit Dial Int. 2008 Mar-Apr;28(2):197-9.

4 McCormick BB, Bargman JM. Noninfectious complications of peritoneal dialysis: implications for patient and technique survival. J Am Soc Nephrol. 2007 Dec;18(12):3023-5.

5 Diaz-Buxo JA. Management of peritoneal catheter malfunction. Perit Dial Int. 1998 May-Jun;18(3):256-9. 
$6 \mathrm{Xu}$ Y, Gao C, Xu J, Chen N. Successful Treatment of Idiopathic Eosinophilic Peritonitis by Oral Corticosteroid Therapy in a Continuous Ambulatory Peritoneal Dialysis Patient. Case Rep Nephrol Dial. 2015 Jun;5(2):130-4.

7 Lee S, Schoen I. Eosinophilia of peritoneal fluid and peripheral blood associated with chronic peritoneal dialysis. Am J Clin Pathol. 1967 May;47(5):638-40.

8 Ejaz AA. Peritoneal fluid eosinophilia. Nephrol Dial Transplant. 1998 Oct;13(10):2463-4.

9 Thakur SS, Unikowsky B, Prichard S. Eosinophilic peritonitis in CAPD: treatment with prednisone and diphenhydramine. Perit Dial Int. 1997 Jul-Aug;17(4):402-3.

10 Fontán MP, Rodríguez-Carmona A, Galed I, Iglesias P, Villaverde P, García-Ureta E. Incidence and significance of peritoneal eosinophilia during peritoneal dialysis-related peritonitis. Perit Dial Int. 2003 Sep-Oct;23(5): $460-4$.

11 Chan MK, Chow L, Lam SS, Jones B. Peritoneal eosinophilia in patients on continuous ambulatory peritoneal dialysis: a prospective study. Am J Kidney Dis. 1988 Feb;11(2):180-3.

12 Jo YI, Shin SK, Song JO. Clinical characteristics of peritoneal fluid eosinophilia in patients on CAPD. Korean J Nephrol. 2003;22:219-27.

13 Oh SY, Kim H, Kang JM, Lim SH, Park HD, Jung SS, et al. Eosinophilic peritonitis in a patient with continuous ambulatory peritoneal dialysis (CAPD). Korean J Intern Med. 2004 Jun;19(2):121-3.

14 Rosner MH, Chhatkuli B. Vancomycin-related eosinophilic peritonitis. Perit Dial Int. 2010 Nov-Dec;30(6): $650-2$.

15 Wang HH, Yang LY, Chang JW, Hung YT, Lee TY, Tang RB. Eosinophilic peritonitis: an unusual manifestation of tuberculous peritonitis in peritoneal dialysis patient. J Chin Med Assoc. 2011 Jul;74(7):322-4.

16 Lee SH, Huang TS. Persistent eosinophilic peritonitis associated with fungal infection cured by resection of external Tenckhoff catheter: a case report. Perit Dial Int. 1997 Jul-Aug;17(4):397-9.

17 Tang S, Lo CY, Lo WK, Chan TM. Resolution of eosinophilic peritonitis with ketotifen. Am J Kidney Dis. 1997 Sep;30(3):433-6. 